\title{
Stochastic evolutionary cartel formation
}

\section{Darong DAI}

Nanjing University, Nanjing

daidarong998@163.com

Kunrong SHEN

Nanjing University, Nanjing

shenkr@nju.edu.cn

\begin{abstract}
This paper employs the evolutionary dynamics driven by the Moran process (a special birth-death process) to investigate endogenous cartel formation from the perspective of stochastic evolution of the underlying industry. A Prisoner's Dilemma game is derived based on the Cournot competition between any two firms. Moreover, in a repeated setting, we consider the normal-form game between two well-known behavior modes: cooperative strategy tit-for-tat (TFT) and non-cooperative strategy always defect (ALLD). We then give the corresponding conditions under which full collusion and partial collusion are established, respectively, in stochastic evolutionary sense. Finally, both the threshold of discount factor and the threshold of industry concentration are endogenously determined in the model.
\end{abstract}

Keywords: Endogenous cartel, Cournot competition, Prisoner's Dilemma, Repeated game, Evolutionary game theory.

JEL Classification: L13, C73.

REL Classification: $1 \mathrm{~J}$. 


\section{Introduction}

When regarding cartel formation, existing literatures (Compte et al., 2002; Vasconcelos, 2005; Escrihuela-Villar, 2009; and Bos and Harrington, 2010) usually assume that the collusive outcome is the joint profit maximum. As you can see, they endogenize cartel composition and cartel formation by using the classic methodology of rational individual choice. That is, in a repeated setting, collusion can be supported and sustained if the expected cost of cheating today is higher than the expected benefit in future, so that firms refrain from undercutting the collusive price. Nevertheless, it is easily seen that bounded rationality and uncertainty in reality indeed may make collusion difficult, particularly in a changing economic environment, because of increased complexity in formulating and monitoring any contract (Williamson, 1975; Levenstein and Suslow, 2006). So, what are the industry conditions which support stochastic evolutionary cartel formation? Are there any new problems or issues when we consider cartel formation in an evolutionary environment and also from the perspective of stochastic group evolution ? $^{(1)}$ The objective of this article is to address these and related questions by endogenizing cartel formation in the context of an evolutionary game with an exogenous parameter measuring the extent of substitution between the products.

The major results of the current exploration can be expressed as follows. First, the recognition that "entry" is one of the most important problems facing any cartel encourages us to employ birth-death processes to model such kind of phenomenon in reality, and in particular, the well-known (perturbed) Moran process is used in this study for the first time. Second, noting that "coordination" plays a key role in cartel success in a situation in which there are multiple equilibria, we thus derive a coordination game based upon the Prisoner's Dilemma game between any two firms and also in a repeated setting, and thus the following analysis strictly surrounds the induced normal-form game. Third, the underlying evolutionary mechanism reveals that "cheating" can be an evolutionary stable strategy (ESS) in relatively weak conditions and therefore cartel formation is faced with much stronger challenge in the present industrial environment. Forth, the minimum discount factor for sustaining full collusion is obtained and we further derive the minimum number of the firms (or the threshold of industry concentration) that supports full collusion for any given discount factor. Finally, we have derived the minimum discount factor such that partial collusion is established when the number of the firms in the underlying industry is sufficiently large and the mutations of the evolutionary dynamics are sufficiently small.

Now, we discuss some related literatures. Selten (1973) has theoretically considered the process of forming a cartel in a simultaneous-moves game in the 
sense of Cournot-competition. The main finding of Selten (1973) is that only the industries composed of less than six firms stand a chance of having a cartel. To extend the model of Selten (1973), Prokop (1999) constructs a sequential- and simultaneous-moves game to investigate the process of cartel formation. In the model with sequential moves Prokop (1999) finds that there is a possibility for creation of a dominant cartel, whereas the simultaneous-moves game shows that it might be close to impossible to form a stable cartel even though one can prove the theoretical existence of such a cartel. Recently, there is a pair of articles which endogenize cartel composition and cartel membership in the context of an infinitely repeated game. For example, Escrihuela-Villar (2009) examines an infinitely repeated quantity setting with identical firms and homogeneous goods and the main result reveals that firms only have an incentive to form the smallest sustainable cartel. In addition to that, Bos and Harrington (2010)'s model is the first to endogenize the composition of a cartel in the context of an infinitely repeated game with heterogeneous firms. And their analysis demonstrates that there exists a stable cartel involving the largest firms when firms are sufficiently patient, while a firm with sufficiently small capacity is not a member of any stable cartel.

When compared with existing work, there are some differences which need be specifically pointed out. First, the most obvious difference is that we endogenize cartel formation from the viewpoint of stochastic group evolution rather than the firm's rational individual choice even though we generally can understand the evolutionary outcome by applying the well-known as if hypothesis from the perspective of ex post. Second, in spite of the firms are assumed to be in repeated interactions, the infinitely repeated game just provides us with a normal-form game and our major analysis follows from the underlying evolutionary dynamics deduced by the normal-form game. That is, we consider a stochastic and dynamic environment instead of a static and deterministic framework. Third, the collusive outcome in the paper is not the joint profit maximum as in Escrihuela-Villar (2008) and among others but a long-run equilibrium selected by the underlying evolutionary mechanism. Forth, asymmetry between the firms characterized by the parameter measuring the extent of substitution between the products does not directly affect the process of cartel formation in the present evolutionary model, and it only indirectly determines the minimum discount factor and the minimum number of firms which support full collusion in the industry under consideration. Last but not least, it is particularly worth emphasizing that the endogenous partial collusion is characterized in the sense of limiting probability distribution and also in asymptotic sense in the present stochastic evolutionary game. Accordingly, these differences may also be regarded as the innovations of the current investigation. 
The article is organized as follows. In section 2, the basic model is described and a normal-form game in a repeated setting is established. In section 3, we first introduce the necessary mathematical background, i.e., the Moran process and the perturbed Moran process, and two key theorems are derived, based upon which we then obtain the main results of the present exploration. Section 4 closes this study with some concluding remarks. Major mathematical derivations appear in Appendix A.

\section{The induced normal-form games}

In the present section, it is assumed that there are two firms in the market. We consider the following inverse demand functions,

$$
p_{i}=\alpha-\beta\left(q_{i}+\theta q_{j}\right), i \neq j, i, j=1,2 .
$$

for $\alpha, \beta>0$ and $0 \leq \theta \leq 1$. The parameter $\theta$ measures the extent of substitution between the products $i$ and $j$. In particular, $\theta=0$ implies that products $i$ and $j$ are completely heterogeneous products while $\theta=1$ showing us that products $i$ and $j$ are homogeneous, i.e., products $i$ and $j$ will completely substitute each other when $\theta=1$. Noting that the parameter $\theta$ will be the unique parameter that characterizes the difference between products $i$ and $j$, it will play a crucial role in the following analysis.

Consider a single-period game. Suppose that the firms play non-cooperatively, each firm solves the following optimization problem by making use of (1),

$$
\max _{q_{i}} \pi_{i}\left(q_{i}, q_{j}\right)=q_{i}\left[\alpha-\beta\left(q_{i}+\theta q_{j}\right)\right]-c q_{i}(i \neq j, i, j=1,2) .
$$

in which $c>0$ denotes the fixed marginal cost. From the first-order conditions of the maximization problem, it can be concluded that the reaction functions are given by,

$$
q_{i}\left(q_{j}\right)=\frac{\alpha-c-\beta \theta q_{j}}{2 \beta}(i \neq j, i, j=1,2) .
$$

Now, by symmetry and some necessary algebra, we get that in the unique Cournot-Nash equilibrium of the one short game, firms' outputs and individual profits are written as follows,

$q_{1}^{*}=q_{2}^{*}=\frac{\alpha-c}{\beta(2+\theta)}, \pi_{1}^{\text {Cournot }}\left(q_{1}^{*}, q_{2}^{*}\right)=\pi_{2}^{\text {Cournot }}\left(q_{1}^{*}, q_{2}^{*}\right)=\frac{(\alpha-c)^{2}}{\beta(2+\theta)^{2}}$. 
Suppose instead that the firms are encouraged to maximize their joint profit in the one-short game, thus their equilibrium outputs result from the following optimization problem,

$$
\max _{\left\{q_{1}, q_{2}\right\}} \sum_{i=1}^{2} \pi_{i}\left(q_{1}, q_{2}\right)=\left\{q_{1}\left[\alpha-\beta\left(q_{1}+\theta q_{2}\right)\right]-c q_{1}\right\}+\left\{q_{2}\left[\alpha-\beta\left(q_{2}+\theta q_{1}\right)\right]-c q_{2}\right\} .
$$

From the first-order conditions of the previous maximization problem, and by symmetry between the two firms, it is easily shown that the cooperative outputs and cooperative-equilibrium profits are given as,

$$
\hat{q}_{1}=\hat{q}_{2}=\frac{\alpha-c}{2 \beta(1+\theta)}, \pi_{1}^{\text {Cooperate }}\left(\hat{q}_{1}, \hat{q}_{2}\right)=\pi_{2}^{\text {Cooperate }}\left(\hat{q}_{1}, \hat{q}_{2}\right)=\frac{(\alpha-c)^{2}}{4 \beta(1+\theta)} .
$$

We without loss of generality assume that firm 1 is considering to deviate when the two firms are supposed to set outputs in (6), then making use of firm 1's reaction function in (3), firm 1's optimal deviation output is obtained,

$$
q_{1}^{*}\left(\hat{q}_{2}\right)=\frac{(2+\theta)(\alpha-c)}{4 \beta(1+\theta)} .
$$

So, the deviation profit of firm 1 and the corresponding cooperative profit of firm 2 are respectively given as,

$$
\begin{aligned}
& \pi_{1}^{\text {Defect }}\left(q_{1}^{*}\left(\hat{q}_{2}\right), \hat{q}_{2}\right)=\frac{1}{\beta}\left[\frac{(2+\theta)(\alpha-c)}{4(1+\theta)}\right]^{2} . \\
& \pi_{2}^{\text {Cooperate }}\left(q_{1}^{*}\left(\hat{q}_{2}\right), \hat{q}_{2}\right)=\frac{(\alpha-c)^{2}\left(2+2 \theta-\theta^{2}\right)}{8 \beta(1+\theta)^{2}} .
\end{aligned}
$$

Up to the present step, we have derived all the possible profits of the two firms by applying the symmetry between the firms. Accordingly, one can establish the payoff matrix of the one-short game by using (4), (6), (8) and (9) as follows,

\begin{tabular}{l|lc} 
& Cooperate & Defect \\
\hline Cooperate & $\frac{(\alpha-c)^{2}}{4 \beta(1+\theta)}$ & $\frac{(\alpha-c)^{2}\left(2+2 \theta-\theta^{2}\right)}{8 \beta(1+\theta)^{2}}$ \\
Defect & $\frac{1}{\beta}\left[\frac{(2+\theta)(\alpha-c)}{4(1+\theta)}\right]^{2}$ & $\frac{(\alpha-c)^{2}}{\beta(2+\theta)^{2}}$
\end{tabular}

Figure 1. One-Short Game 
Now, based upon the one-short normal-form game given in Figure 1, we obtain the following result,

Lemma 1. i) If $\theta=0$, i.e., the products of the two firms are completely heterogeneous, then Figure 1 shows us a Neutral game with the entries having the same value of $(\alpha-c)^{2} / 4 \beta$;

ii) If $\theta \in[0,1] \cap\left\{\theta \mid 8(1+\theta)^{2}>(2+\theta)^{2}\left(2+2 \theta-\theta^{2}\right)\right\} \neq \varnothing$, then Figure 1 shows a Prisoners' Dilemma game with

$\pi_{1}^{\text {Defect }}\left(q_{1}^{*}\left(\hat{q}_{2}\right), \hat{q}_{2}\right)>\pi_{1}^{\text {Cooperate }}\left(\hat{q}_{1}, \hat{q}_{2}\right)>\pi_{1}^{\text {Cournot }}\left(q_{1}^{*}, q_{2}^{*}\right)>\pi_{1}^{\text {Cooperate }}\left(\hat{q}_{1}, q_{2}^{*}\left(\hat{q}_{1}\right)\right)$ for firm 1 , and so is firm 2 .

Proof. Inserting $\theta=0$ into Figure 1 produces the required result in i). It follows from Figure 1 that,

$\pi_{1}^{\text {Cournot }}\left(q_{1}^{*}, q_{2}^{*}\right)>\pi_{1}^{\text {Cooperate }}\left(\hat{q}_{1}, q_{2}^{*}\left(\hat{q}_{1}\right)\right) \Leftrightarrow 8(1+\theta)^{2}>(2+\theta)^{2}\left(2+2 \theta-\theta^{2}\right)$

Indeed, we have $0 \notin[0,1] \cap\left\{\theta \mid 8(1+\theta)^{2}>(2+\theta)^{2}\left(2+2 \theta-\theta^{2}\right)\right\} \neq \varnothing$, for example, one may check it with $\theta=1$. Noting that,

$$
\frac{(\alpha-c)^{2}}{4 \beta(1+\theta)}<\frac{1}{\beta}\left[\frac{(2+\theta)(\alpha-c)}{4(1+\theta)}\right]^{2} \Leftrightarrow-\theta^{2}<0
$$

we obtain $\pi_{1}^{\text {Defect }}\left(q_{1}^{*}\left(\hat{q}_{2}\right), \hat{q}_{2}\right)>\pi_{1}^{\text {Cooperate }}\left(\hat{q}_{1}, \hat{q}_{2}\right)$ for $\theta \in(0,1]$. Moreover, we also get $\pi_{1}^{\text {Cooperate }}\left(\hat{q}_{1}, \hat{q}_{2}\right)>\pi_{1}^{\text {Cournot }}\left(q_{1}^{*}, q_{2}^{*}\right)$ for $\theta \in(0,1]$ as we have,

$\frac{(\alpha-c)^{2}}{4 \beta(1+\theta)}>\frac{(\alpha-c)^{2}}{\beta(2+\theta)^{2}} \Leftrightarrow \theta^{2}>0$

so, the results in ii) are derived.

In what follows, we will consider the infinitely repeated game between the two firms. We let $0<\delta<1$ denote the common discount factor. In order to make the notations more compact, we put,

$$
\begin{aligned}
& \xi_{1} \equiv \frac{(\alpha-c)^{2}}{4 \beta(1+\theta)}, \xi_{2} \equiv \frac{(\alpha-c)^{2}\left(2+2 \theta-\theta^{2}\right)}{8 \beta(1+\theta)^{2}} . \\
& \xi_{3} \equiv \frac{1}{\beta}\left[\frac{(2+\theta)(\alpha-c)}{4(1+\theta)}\right]^{2}, \xi_{4} \equiv \frac{(\alpha-c)^{2}}{\beta(2+\theta)^{2}} .
\end{aligned}
$$


Indeed, we will focus on the following two kinds of well-known behavior mode: TFT (tit-for-tat) and ALLD (always defect). Thus, based on Figure 1, we obtain the following normal-form game by using (10) and (11).

\begin{tabular}{l|ll} 
& TFT & ALLD \\
\hline TFT & $\frac{\xi_{1}}{1-\delta}$ & $\frac{\xi_{2}+\left(\xi_{4}-\xi_{2}\right) \delta}{1-\delta}$ \\
ALLD & $\frac{\xi_{3}+\left(\xi_{4}-\xi_{3}\right) \delta}{1-\delta}$ & $\frac{\xi_{4}}{1-\delta}$
\end{tabular}

Figure 2. Infinitely Repeated Game

By Figure 2 and Lemma 1, the following lemma can be easily established.

Lemma 2. It is assumed that Figure 1 provides us with a Prisoners' Dilemma game, i.e., $\xi_{3}>\xi_{1}>\xi_{4}>\xi_{2}$ in (10) and (11). If $0<\delta<\frac{\xi_{3}-\xi_{1}}{\xi_{3}-\xi_{4}},\{A L L D, A L L D\}$ will be the unique Nash equilibrium; if $\frac{\xi_{3}-\xi_{1}}{\xi_{3}-\xi_{4}} \leq \delta<1$, then there are two pure-strategy Nash equilibria $\{T F T, T F T\}$ and $\{A L L D, A L L D\}$, a mixed-strategy Nash equilibrium represented by $\{(\gamma, 1-\gamma),(\gamma, 1-\gamma)\}$ in which

$$
\gamma=\frac{\left(\xi_{4}-\xi_{2}\right)(1-\delta)}{\left(\xi_{1}-\xi_{2}-\xi_{3}+\xi_{4}\right)-\left(2 \xi_{4}-\xi_{2}-\xi_{3}\right) \delta},
$$

and we also get that

$$
\frac{\xi_{1}}{1-\delta}>\frac{\xi_{3}+\left(\xi_{4}-\xi_{3}\right) \delta}{1-\delta}>\frac{\xi_{4}}{1-\delta}>\frac{\xi_{2}+\left(\xi_{4}-\xi_{2}\right) \delta}{1-\delta}
$$

Proof. Noting that the corresponding proof is quite easy and hence we take it omitted.

Furthermore, by applying Lemma 2, we obtain the following result,

Lemma 3. It is assumed that Figure 1 provides us with a Prisoners' Dilemma game, i.e., $\xi_{3}>\xi_{1}>\xi_{4}>\xi_{2}$ in (10) and (11), and also we let $\frac{\xi_{3}-\xi_{1}}{\xi_{3}-\xi_{4}} \leq \delta<1$, i.e., Figure 2 is indeed a coordination game. Then it is easily seen that $\{T F T, T F T\}$ Pareto (payoff) dominates $\{A L L D, A L L D\}$. Moreover, if $\frac{\xi_{3}-\xi_{1}+\xi_{4}-\xi_{2}}{\xi_{3}-\xi_{2}}<\delta<1$, we get that $\left\{\right.$ TFT, TFT\} risk dominates $\{A L L D, A L L D\}$; if $\frac{\xi_{3}-\xi_{1}}{\xi_{3}-\xi_{4}} \leq \delta<\frac{\xi_{3}-\xi_{1}+\xi_{4}-\xi_{2}}{\xi_{3}-\xi_{2}}$, then we have that $\{A L L D, A L L D\}$ risk dominates $\{T F T, T F T\}$. 
Proof. It is widely known that $\{$ TFT, TFT $\}$ (strictly) risk dominates $\{$ ALLD, ALLD $\}$ if and only if,

$$
\frac{\xi_{1}}{1-\delta}-\frac{\xi_{3}+\left(\xi_{4}-\xi_{3}\right) \delta}{1-\delta}>\frac{\xi_{4}}{1-\delta}-\frac{\xi_{2}+\left(\xi_{4}-\xi_{2}\right) \delta}{1-\delta}
$$

which implies that,

$$
\frac{\xi_{3}-\xi_{1}+\xi_{4}-\xi_{2}}{\xi_{3}-\xi_{2}}<\delta<1
$$

Moreover, noting that,

$$
\frac{\xi_{3}-\xi_{1}}{\xi_{3}-\xi_{4}}>\frac{\xi_{3}-\xi_{1}+\xi_{4}-\xi_{2}}{\xi_{3}-\xi_{2}} \Leftrightarrow\left(\xi_{4}-\xi_{2}\right)\left(\xi_{4}-\xi_{1}\right)>0
$$

which contradicts with the assumption that Figure 1 provides us with a Prisoners' Dilemma game. Therefore, we get,

$$
\frac{\xi_{3}-\xi_{1}}{\xi_{3}-\xi_{4}}<\frac{\xi_{3}-\xi_{1}+\xi_{4}-\xi_{2}}{\xi_{3}-\xi_{2}}
$$

which gives the required result.

Why do we focus on the case that Figure 2 defines a coordination game? As argued by Tirole (1988), Martin (2002) and Levenstein and Suslow (2006), coordination is one of the most important problems facing any cartel and firms must be able to coordinate on an equilibrium in a situation in which there are often multiple equilibria. In other words, coordination game will provide us with a much better approximation to reality and it certainly implies much richer economic implications and this is why we directly suppose throughout that both TFT and ALLD are (strict) Nash-equilibrium strategies in Figure 2. And as you can see below, we mainly employ the well-known evolutionary mechanism in solving the present equilibrium-selection problem facing the firms in the underlying industry.

\section{Evolutionary characterization of cartel formation}

\subsection{Frequency dependent processes}

Now, we will introduce the stochastic evolutionary game dynamics in finite populations that is quite similar to the well-known replicator dynamics for infinite populations. In particular, we will employ the Moran process that is well known in population genetics (Ewens, 2004) where it is normally used to study the 
dynamics of constant selection in finite populations. Indeed, the Moran process describes a biological population with asynchronous reproduction, that is, a single individual is chosen to reproduction at any one time step. Thus, there would be overlapping generations in the process, which accordingly provides us with a possible approximation to the characteristic of the evolution of firms in any given industry. For example, fierce market competition implies that there are new entrants as well as incumbent firms for some given time interval and in most of the industries of the market economy. Provided that the time step of reproduction is sufficiently small, we may argue that the Moran process will be a suitable tool for our analysis of the evolutionary characterization of firm's strategic behaviors. It is especially worthwhile mentioning that the results in the current section are directly brought from Taylor et al. (2004), Nowak et al. (2004) and Fudenberg et al. (2006).

\subsubsection{The Moran process}

Let us consider a symmetric game between two strategies, A and B, with payoff matrix,

\begin{tabular}{l|ll} 
& $\mathrm{A}$ & $\mathrm{B}$ \\
\hline $\mathrm{A}$ & $a_{11}$ & $a_{12}$ \\
$\mathrm{~B}$ & $a_{21}$ & $a_{22}$
\end{tabular}

Figure 3. $a_{11}>a_{21}>a_{22}>a_{12}$

The fitness of strategies A and B with payoff matrix in Figure 3 is, respectively, given by,

$$
f_{i}=1-w+\frac{w\left[a_{11}(i-1)+a_{12}(N-i)\right]}{N-1}, g_{i}=1-w+\frac{w\left[a_{21} i+a_{22}(N-i-1)\right]}{N-1}
$$

in which $i$ denotes the number of individuals using strategy A, and $w \in[0,1]$ specifies the contribution of the game to the fitness. This parameter $w$, quantifying the intensity of selection, cancels out in deterministic replicator dynamics of infinite populations, but plays a crucial role in finite populations (e.g., Nowak et al., 2004; Fudenberg et al., 2006; Imhof and Nowak, 2006). Let us compare $f_{i}$ and $g_{i}$ for each $i$ in order to evaluate whether selection acts to increase or reduce the number of A players at position $i$. Put,

$$
h_{i} \equiv f_{i}-g_{i}=\frac{w}{N-1}\left[a_{11}(i-1)+a_{12}(N-i)-a_{21} i-a_{22}(N-i-1)\right]
$$


which yields that $h_{i}$ is a linear function of $i$ defined on $i=1, \ldots, N-1$. Invasion dynamics can be characterized by evaluating the sign of $h_{1}$ and $h_{N-1}$. If $h_{1}>0$ then we say "selection favors A invading B". If $h_{N-1}<0$ then we say "selection favors B invading A". These invasion criteria evaluate whether a single individual of A (or B) has a higher fitness than the resident population.

At each time step, an individual is chosen for reproduction proportional to its fitness. One identical offspring is being produced which replaces another randomly chosen individual. Thus the population size, $N$, is strictly constant. And at each time step, the number of A individuals can either increase by one, stay the same or fall by one. Consequently, the transition matrix of the Markov process is tri-diagonal and defines a birth-death process. The transition matrix is given by,

$$
P_{i, i+1}=\frac{i f_{i}}{i f_{i}+(N-i) g_{i}} \frac{N-i}{N}, P_{i, i-1}=\frac{(N-i) g_{i}}{i f_{i}+(N-i) g_{i}} \frac{i}{N}, P_{i, i}=1-P_{i, i+1}-P_{i, i-1}
$$

all other entries of the transition matrix are 0 .

In this paper, we are particularly interested in the probability that a single A individual reaches fixation in a population of $\mathrm{B}$ individuals. This probability is given by,

$$
\rho_{A B}=\frac{1}{1+\sum_{j=1}^{N-1} \prod_{k=1}^{j} \frac{g_{k}}{f_{k}}}
$$

which is derived by Nowak et al. (2004), Fudenberg et al. (2006), and Imhof and Nowak (2006). And if $\rho_{A B}>1 / N$, then selection favors A replacing B. Deterministic replicator dynamics of infinite populations admit an unstable equilibrium at a frequency of $\mathrm{A}$ given by $x^{*}=\left(a_{22}-a_{12}\right) /\left(a_{11}-a_{12}-a_{21}+a_{22}\right)$. If the initial frequency of $\mathrm{A}$ is less than $x^{*}$, then it will be eliminated by natural selection. A can only replace B if its initial frequency exceeds this invasion barrier. The following theorem and definition are established by Nowak et al. (2004).

Theorem 1. For a given population size $N$ and sufficiently weak selection (small $w)$, selection favors $A$ replacing $B$ if $a_{11}(N-2)+a_{12}(2 N-1)>a_{21}(N+1)+$ $a_{22}(2 N-4)$. For the smallest possible population size, $N=2$, we obtain $a_{12}>a_{21}$. For the limit of large $N$, we obtain $a_{11}+2 a_{12}>a_{21}+2 a_{22}$, which is equivalent to $x^{*}<1 / 3$. Moreover, if $x^{*}<1 / 3$, then there is a minimum $N$, i.e., 
$N_{\min } \equiv\left(2 a_{11}+a_{12}+a_{21}-4 a_{22}\right) /\left(a_{11}+2 a_{12}-a_{21}-2 a_{22}\right)$

such that $\rho_{A B}>1 / N$.

Definition 1. (Evolutionary Stability). For finite population size, $N$, we propose that B is an evolutionary stable strategy (ESS) if two conditions hold: (i) selection opposes $\mathrm{A}$ invading $\mathrm{B}$, which means that a single mutant of $\mathrm{A}$ in a population of $\mathrm{B}$ players has a lower fitness; and (ii) selection opposes A replacing $\mathrm{B}$, which means $\rho_{A B}<1 / N$. So, strategy B is ESS if $a_{12}(N-1)<a_{21}+a_{22}(N-2)$ and $a_{11}(N-2)+$ $a_{12}(2 N-1)<a_{21}(N+1)+a_{22}(2 N-4)$. For $N=2$ both conditions thus reduce to $a_{12}<a_{21}$. For large populations, the two conditions lead us to $a_{12}<a_{22}$ and $x^{*}>1 / 3$, respectively.

\subsubsection{Perturbed Moran process}

In this subsection, we will introduce mutation into the Moran process discussed above. That is, we assume that with probability $\mu_{A B}>0$, an A-offspring is a mutant which plays $\mathrm{B}$ instead of $\mathrm{A}$, and with probability $\mu_{B A}>0$, a B-offspring plays A. After reproduction, the offspring replaces a randomly chosen member of the population, so that the population size is constant. The process $X(t)$ that describes the number of individuals that use A is a Markov process with state space $\{0, \ldots, N\}$ and transition matrix $\left(P_{i j}\right)$, where $P_{i j}=0$ if $|i-j|>1$, $P_{01}=\mu_{B A}=1-P_{00}, P_{N, N-1}=\mu_{A B}=1-P_{N N}$ and for $i=1, \ldots, N-1$,

$P_{i, i+1}=\frac{i f_{i}\left(1-\mu_{A B}\right)+(N-i) g_{i} \mu_{B A}}{i f_{i}+(N-i) g_{i}} \frac{N-i}{N}$

$P_{i, i-1}=\frac{i f_{i} \mu_{A B}+(N-i) g_{i}\left(1-\mu_{B A}\right)}{i f_{i}+(N-i) g_{i}} \frac{i}{N}$

$P_{i, i}=1-P_{i, i+1}-P_{i, i-1}$

Now, the following theorem can be established,

Theorem 2. Let $N \rightarrow \infty, \mu_{A B} N \rightarrow \kappa_{1}, \mu_{B A} N \rightarrow \kappa_{2}$ with $0<\kappa_{1}, \kappa_{2}<\infty$. If we have $h_{i}=f_{i}-g_{i}=0$ for $\forall i \in\{0, \ldots ., N\}$, then the limiting distribution of the process $X(t)$ follows a Beta distribution with parameters $\kappa_{1}$ and $\kappa_{2}$ given above.

Proof. See Appendix A. 
One can also refer to Fudenberg and Imhof $(2006,2008)$ for much more general results about the equilibrium selection and limit distribution in such kind of evolutionary dynamics.

\subsection{Evolutionary analysis on firm's behavior}

Based upon Figure 2, (12) can be written as follows,

$$
h_{i}=\frac{w}{N-1}\left[\frac{\xi_{1}}{1-\delta}(i-1)+\frac{\xi_{2}+\left(\xi_{4}-\xi_{2}\right) \delta}{1-\delta}(N-i)-\frac{\xi_{3}+\left(\xi_{4}-\xi_{3}\right) \delta}{1-\delta} i-\frac{\xi_{4}}{1-\delta}(N-i-1)\right]
$$

So, based on the results in the previous section, we get,

Proposition 1. For the infinitely repeated game defined in Figure 2, selection does not favor TFT invading ALLD. And selection favors ALLD invading TFT if we put $\delta \in\left[\frac{\xi_{3}-\xi_{1}}{\xi_{3}-\xi_{4}}, \frac{\left(\xi_{3}-\xi_{1}\right)(N-1)+\xi_{1}-\xi_{2}}{\left(\xi_{3}-\xi_{4}\right)(N-1)+\xi_{4}-\xi_{2}}\right)$.

Proof. By using (16), it is easily shown that $h_{1}>0 \Leftrightarrow \delta>1$. This implies that selection does not favor TFT invading ALLD. Also by (16), we get,

$$
h_{N-1}<0 \Leftrightarrow \delta<\frac{\left(\xi_{3}-\xi_{1}\right)(N-1)+\xi_{1}-\xi_{2}}{\left(\xi_{3}-\xi_{4}\right)(N-1)+\xi_{4}-\xi_{2}}
$$

And it is demonstrated that,

$$
\frac{\left(\xi_{3}-\xi_{1}\right)(N-1)+\xi_{1}-\xi_{2}}{\left(\xi_{3}-\xi_{4}\right)(N-1)+\xi_{4}-\xi_{2}} \geq \frac{\xi_{3}-\xi_{1}}{\xi_{3}-\xi_{4}} \Leftrightarrow\left(\xi_{1}-\xi_{2}\right)\left(\xi_{3}-\xi_{4}\right) \geq\left(\xi_{3}-\xi_{1}\right)\left(\xi_{4}-\xi_{2}\right)
$$

which always holds due to the fact $\xi_{3}>\xi_{1}>\xi_{4}>\xi_{2}$ of Figure 1 . Hence, the proof is complete.

Although it is assumed that both ALLD and TFT are (strict) Nash-equilibrium strategies in Figure 2, Proposition 1 proves that stochastic evolutionary selection favors ALLD invading TFT, i.e., a single ALLD-firm has a higher fitness than the resident TFT-firms, when the discount factor is strictly less than $\frac{\left(\xi_{3}-\xi_{1}\right)(N-1)+\xi_{1}-\xi_{2}}{\left(\xi_{3}-\xi_{4}\right)(N-1)+\xi_{4}-\xi_{2}} \equiv \delta_{\text {sup }}$. As you can see, $\delta_{\text {sup }}$ strictly relies on the number of firms in the industry under consideration (or the industry concentration), the parameter $\theta$ that measures the extent of substitution between the products (or asymmetry between the products or firms) and also the fixed marginal cost $c$ by noting the definitions given in (10) and (11). Furthermore, Proposition 1 also demonstrates a robust claim that selection driven by the Moran process does not favor TFT invading ALLD, i.e., a single TFT- firm can never have a fitness higher than the 
resident ALLD-firms, even if TFT both payoff and risk dominates ALLD (making use of Lemma 3). To sum up, Proposition 1 highlights the great incentive to cheat facing the firms from the viewpoint of stochastic group evolution, thereby undermining the firms' attempts to collude when we employ the classic as if hypothesis for the firms in the industry and also from the ex post perspective.

Proposition 2. (Full Collusion). If there are only two firms in the industry, we obtain that selection does not favor TFT replacing ALLD. For the limit of large $N$, selection favors TFT replacing $A L L D$ if $\delta>\max \left\{\frac{\xi_{3}-\xi_{1}}{\xi_{3}-\xi_{4}}, \frac{2\left(\xi_{4}-\xi_{2}\right)+\xi_{3}-\xi_{1}}{\xi_{3}+\xi_{4}-2 \xi_{2}}\right\}$.

Furthermore, there is a minimum number of firms,

$N_{\min }=\frac{\left(2 \xi_{1}+\xi_{2}+\xi_{3}-4 \xi_{4}\right)+\left(2 \xi_{4}-\xi_{2}-\xi_{3}\right) \delta}{\left(\xi_{1}+2 \xi_{2}-\xi_{3}-2 \xi_{4}\right)+\left(\xi_{4}-2 \xi_{2}+\xi_{3}\right) \delta}$

such that selection favors TFT replacing ALLD.

Proof. Combining Theorem 1 with Figure 2 easily confirms the desired results. In particular, by (16) and Lemma 2,

$\gamma<\frac{1}{3} \Leftrightarrow \delta>\frac{2\left(\xi_{4}-\xi_{2}\right)+\xi_{3}-\xi_{1}}{\xi_{3}+\xi_{4}-2 \xi_{2}}$

and also,

$\frac{2\left(\xi_{4}-\xi_{2}\right)+\xi_{3}-\xi_{1}}{\xi_{3}+\xi_{4}-2 \xi_{2}}<1 \Leftrightarrow \xi_{4}<\xi_{1}$

which holds because Figure 1 is assumed to be a Prisoner's Dilemma game. Thus, the proof is complete.

Proposition 2 establishes the explicit conditions based on which stochastic evolutionary selection driven by the Moran process favors full collusion in the underlying industry, thereby leading us to the endogenous cartel formation. As a matter of fact, full collusion can be reached as long as there are sufficiently many firms in the industry and the firms are sufficiently patient. And we have further derived the closed-form solutions of the minimum number of firms (it depends on discount factor $\delta$, the parameter $\theta$ measuring the extent of substitution between the products and the fixed marginal cost $c$ ) and the minimum discount factor (it relies on the parameter $\theta$ measuring the extent of substitution between the products and the fixed marginal cost $c$ ) so that full collusion is identified in the industry under consideration and also in stochastic evolutionary sense.

What is the corresponding economic intuition of the results shown in Proposition 2? First, in a repeated setting, the threshold discount factor in Proposition 2 
implies that as if the firm in the industry weighs the expected gain from cheating today with the expected reduction or cost of cheating in future even from the perspective of group-level stochastic evolution rather than rational individual choice. Second, the number of firms discussed in Proposition 2 naturally leads us to the crucial concept of industry concentration and the threshold $N_{\min }$ in Proposition 2 sufficiently captures the tradeoff between the following two opposite effects in stochastic evolutionary sense: on the one hand, an increase in concentration increases each individual firm's payoff from collusion, thereby facilitating collusion as argued by early Structure- Conduct-Performance theorists such as Bain $(1951,1956,1959)$, while on the other hand, the value of each firm's share of collusive industry profits declines as the number of firms in the industry increases. That is, in order for firms to be as if willing to refrain from cheating in the present evolutionary environment, there must be a critical value of the number of firms provided any value of the discount factor, any value of the marginal cost and any value of the extent of asymmetry between the products or firms. In addition to that, as emphasized by Sutton $(1991,1998)$ and Symeonidis (2002) that concentration is not exogenous to the history of collusion in the industry, industry concentration is indeed endogenously determined in Proposition 2. And it would be regarded as a long-run equilibrium by noting that the present exploration focuses on evolutionary analysis of industrial organizations.

Proposition 3. If there are two firms in the industry, then ALLD is an evolutionary stable strategy (ESS) in the sense of Definition 1. Moreover, if there is a large number of firms in the industry, then ALLD is an evolutionary stable strategy (ESS) when $\delta \in\left[\frac{\xi_{3}-\xi_{1}}{\xi_{3}-\xi_{4}}, \frac{2\left(\xi_{4}-\xi_{2}\right)+\xi_{3}-\xi_{1}}{\xi_{3}+\xi_{4}-2 \xi_{2}}\right)$.

Proof. Using Definition 1, Figure 2 and Proposition 2 confirms the required assertion. Here we just check that $\frac{\xi_{3}-\xi_{1}}{\xi_{3}-\xi_{4}}<\frac{2\left(\xi_{4}-\xi_{2}\right)+\xi_{3}-\xi_{1}}{\xi_{3}+\xi_{4}-2 \xi_{2}}$. In fact,

$$
\begin{aligned}
& \frac{2\left(\xi_{4}-\xi_{2}\right)+\xi_{3}-\xi_{1}}{\xi_{3}+\xi_{4}-2 \xi_{2}}>\frac{\xi_{3}-\xi_{1}}{\xi_{3}-\xi_{4}} \\
& \Leftrightarrow 2\left(\xi_{4}-\xi_{2}\right)\left(\xi_{3}-\xi_{4}\right)+\left(\xi_{3}-\xi_{1}\right)\left(\xi_{3}-\xi_{4}\right)>\left(\xi_{3}-\xi_{1}\right)\left(\xi_{3}+\xi_{4}-2 \xi_{2}\right) \\
& \Leftrightarrow 2\left(\xi_{4}-\xi_{2}\right)\left(\xi_{3}-\xi_{4}\right)>2\left(\xi_{3}-\xi_{1}\right)\left(\xi_{4}-\xi_{2}\right) \\
& \Leftrightarrow \xi_{1}>\xi_{4}
\end{aligned}
$$

which always holds true since we have supposed that Figure 1 provides us with a Prisoner's Dilemma game, i.e., $\xi_{3}>\xi_{1}>\xi_{4}>\xi_{2}$. As a consequence, the proof is complete. 
If there are only two firms in the industry, then the non-cooperative strategy ALLD will be an ESS, that is, a single mutant of TFT-firm in a group of ALLDfirm has a lower fitness and stochastic evolutionary selection opposes the cooperative strategy TFT replacing the non-cooperative strategy ALLD. Moreover, if there are a large number of firms in the industry, then the noncooperative strategy ALLD will be an ESS only when certain requirement is imposed on the discount factor. Indeed, we have derived the threshold $\delta_{\text {sup }} \equiv \frac{2\left(\xi_{4}-\xi_{2}\right)+\xi_{3}-\xi_{1}}{\xi_{3}+\xi_{4}-2 \xi_{2}}$, which is endogenously determined by the important parameters $\theta$ and $c$ owing to the definitions given by (10) and (11).

Although Proposition 3 focuses on the evolutionary stability of the noncooperative strategy ALLD, it is especially worth emphasizing that it is not necessarily implying that the cooperative strategy TFT is evolutionary unstable. So, what's the underlying implication of Proposition 3? As argued by the classic article of Stigler (1964), Proposition 3 highlights potential cheating as the preeminent challenge that cartels face in repeated interactions and also in stochastic evolutionary sense. In fact, Proposition 3 strengths the argument of Stigler by identifying that cheating can be evolutionary stale under relatively weak conditions. To summarize, Proposition 3 yields that cartel formation faces much stronger challenge in the present stochastic evolutionary environment.

Proposition 4. (Partial Collusion). There exists $\delta \geq \frac{\xi_{3}-\xi_{1}}{\xi_{3}-\xi_{4}}$ such that $h_{i}=0$ in (16) for $\forall i \in\{0, \ldots, N\}$. Let $N \rightarrow \infty, \mu_{A B} N \rightarrow \kappa_{1}, \mu_{B A} N \rightarrow \kappa_{2}$ with $0<\kappa_{1}, \kappa_{2}<\infty$, then the limiting distribution of the stochastic process $X(t)$ is a Beta distribution with parameters $\kappa_{1}$ and $\kappa_{2}$ given above.

Proof. We just need to show that there exists $\delta \geq \frac{\xi_{3}-\xi_{1}}{\xi_{3}-\xi_{4}}$ such that $h_{i}=0$ in (16) for $\forall i \in\{0, \ldots, N\}$. And the remaining proof is quite similar to that of Theorem 2. By (16),

$$
h_{i}=0 \Leftrightarrow \delta(i, N)=\frac{\xi_{3} i+\xi_{4}(N-i-1)-\xi_{1}(i-1)-\xi_{2}(N-i)}{\left(\xi_{4}-\xi_{2}\right)(N-i)-\left(\xi_{4}-\xi_{3}\right) i}
$$

Noting that,

$$
\frac{\partial \delta(i, N)}{\partial i}=\frac{\left(\xi_{1}-\xi_{4}\right)\left[\xi_{4}-\xi_{3}-\left(\xi_{4}-\xi_{2}\right)(N-1)\right]}{\left[\left(\xi_{4}-\xi_{2}\right)(N-i)-\left(\xi_{4}-\xi_{3}\right) i\right]^{2}}<0
$$

due to the fact that $\xi_{3}>\xi_{1}>\xi_{4}>\xi_{2}$ in Figure 1. Thus, we get, 
$\min _{N} \min _{i} \delta(i, N)=\lim _{N \rightarrow \infty} \lim _{i \rightarrow N} \delta(i, N)=\frac{\xi_{3}-\xi_{1}}{\xi_{3}-\xi_{4}}$

which shows the required assertion.

Intuitively, Proposition 4 shows that there is a minimum value $\delta_{\min } \equiv \frac{\xi_{3}-\xi_{1}}{\xi_{3}-\xi_{4}}$ (it is especially worth noting from (10) and (11) that this value is endogenously determined by two parameters, i.e., the parameter $\theta$ measuring the extent of substitution between the products and the fixed marginal cost $c$ ) of the discount factor such that neither selection favors ALLD invading TFT nor selection favors TFT invading ALLD in the underlying evolutionary game characterizing the endogenous changes of organizational structures in evolutionary sense. In other words, provided the repeated interactions between the firms and also provided the firms are sufficiently patient, a single ALLD-firm cannot invade into a group of TFT-firms and a single TFT-firm cannot invade into a group of ALLD-firms in stochastic evolutionary sense.

What is more, it is demonstrated in Proposition 4 that the limiting distribution of the Markov process $X(t)$ which represents the number of TFT-firms at time $t$ follows a Beta distribution as long as the firms are sufficiently patient, the number of the firms in the given industry is sufficiently large and the mutations of the underlying evolutionary dynamics are sufficiently small. In spite of full collusion (e.g., all firms employ the cooperative strategy TFT in repeated interactions) may not be established in the present case, we indeed have a explicit characterization of the limiting distribution of the number of colluded firms in the given industry and in the circumstance corresponding to Proposition 4. In other words, such kind of cartel is indexed by the number of the firms that use cooperative strategy TFT in repeated interactions. Meanwhile, it would be easily seen from Proposition 4 that the endogenously formed cartel exhibits asymptotic stability in certain sense.

\section{Concluding remarks}

What are the explicit conditions which support stochastic evolutionary cartel formation? Can we establish full collusion or at least partial collusion under a given evolutionary mechanism? How encompassing is a cartel in evolutionary sense? In spite of the large body of theoretical work on collusion, there is little research that addresses these questions within the evolutionary game theory framework. The objective of our research is to shed some light on these questions and we reexamine some classic arguments about cartel formation in stochastic evolutionary sense, i.e., we employ the methodology of stochastic group evolution rather than the principle of rational individual choice. We find that the issue of 
cartel formation is essentially an equilibrium-selection problem in our model, and also our study focuses on long-run equilibrium due to the characteristics of industrial evolution in reality.

First, it is shown that cheating can be an evolutionary stable strategy (ESS) under relatively weak conditions, and hence cartel formation is faced with much stronger challenge in the present evolutionary framework. Second, we have derived the minimum number of firms in the underlying industry (or the threshold of industry concentration) that supports full collusion in stochastic evolutionary sense. A third finding is that, in a mutation-selection dynamics, there exists a minimum discount factor such that partial collusion can be established when the number of firms is sufficiently large and the mutations are sufficiently small. Finally, it is worthwhile mentioning that the discount factor still plays a crucial role in the current evolutionary model and the corresponding domain is endogenously determined by the parameter measuring the extent of substitution between the products (or asymmetry between the products or firms) and the fixed marginal cost. Noting that we just construct a simple model from the evolutionary perspective, rich extensions can be done based on our framework and we leave it to future work.

\section{Appendix A}

Proof of Theorem 2. Some of the results are brought from Karlin and Taylor (1975, pp.141-144). If $h_{i}=f_{i}-g_{i}=0$ for $\forall i \in\{0, \ldots ., N\}$, then the transition matrix defined by (13)-(15) can be rewritten as follows,

$$
\begin{aligned}
& P_{i, i+1}=\left(1-\frac{i}{N}\right)\left[\frac{i}{N}\left(1-\mu_{A B}\right)+\left(1-\frac{i}{N}\right) \mu_{B A}\right] \\
& P_{i, i-1}=\frac{i}{N}\left[\frac{i}{N} \mu_{A B}+\left(1-\frac{i}{N}\right)\left(1-\mu_{B A}\right)\right] \\
& P_{i, i}=1-P_{i, i+1}-P_{i, i-1}
\end{aligned}
$$

The stochastic process described above is thus a birth and death process with a finite number of states $i \in\{0, \ldots ., N\}$ whose infinitesimal birth and death rates are, respectively, given by,

$$
\lambda_{i} \equiv \lambda\left(1-\frac{i}{N}\right)\left[\frac{i}{N}\left(1-\mu_{A B}\right)+\left(1-\frac{i}{N}\right) \mu_{B A}\right]
$$


$\mu_{i} \equiv \lambda \frac{i}{N}\left[\frac{i}{N} \mu_{A B}+\left(1-\frac{i}{N}\right)\left(1-\mu_{B A}\right)\right]$

in which we have assumed that the probability that the state changes during the time interval $(t, t+\Delta)$ is $\lambda \Delta+o(\Delta)$ independent of the values of $X(t)$ and that the probability of two or more changes occurring in a time interval $\Delta$ is $o(\Delta)$ with some constant $\lambda>0$. So, we obtain,

$$
\begin{aligned}
& \lambda_{i}=\frac{\lambda(N-i)}{N^{2}}\left(1-\mu_{A B}-\mu_{B A}\right) i\left(1+\frac{F}{i}\right) \\
& \mu_{i}=\frac{\lambda(N-i)}{N^{2}}\left(1-\mu_{A B}-\mu_{B A}\right) i\left(1+\frac{H}{N-i}\right)
\end{aligned}
$$

where $F \equiv N \mu_{B A} /\left(1-\mu_{A B}-\mu_{B A}\right)$ and $H \equiv N \mu_{A B} /\left(1-\mu_{A B}-\mu_{B A}\right)$. Put,

$$
\pi_{k} \equiv \frac{\lambda_{0} \lambda_{1} \cdots \lambda_{k-1}}{\mu_{1} \mu_{2} \cdots \mu_{k}}
$$

Then we obtain,

$$
\log \pi_{k}=\sum_{i=1}^{k-1} \log \left(1+\frac{F}{i}\right)-\sum_{i=1}^{k-1} \log \left(1+\frac{H}{N-i}\right)+\log (N F)-\log \left[(N-k) k\left(1+\frac{H}{N-k}\right)\right]
$$

Making use of Taylor expansion and letting $k \rightarrow \infty$,

$$
\begin{aligned}
& \sum_{i=1}^{k-1} \log \left(1+\frac{F}{i}\right) \sim \log \left(k^{F}\right)+\varepsilon_{k} \\
& \sum_{i=1}^{k-1} \log \left(1+\frac{H}{N-i}\right) \sim \log \left[\frac{N^{H}}{(N-k)^{H}}\right]+\varsigma_{k}
\end{aligned}
$$

where $\lim _{k \rightarrow \infty} \varepsilon_{k}<\infty$ and $\lim _{k \rightarrow \infty} \varsigma_{k}<\infty$. Thus,

$\log \pi_{k} \sim \log \left[\eta_{k} \frac{k^{F}(N-k)^{H} N F}{N^{F}(N-k) k}\right], k \rightarrow \infty$

where we let $\lim _{k \rightarrow \infty} \eta_{k} \equiv \lim _{k \rightarrow \infty}\left(\varepsilon_{k}+\varsigma_{k}\right) \equiv \eta$. Noting that $F \rightarrow \kappa_{2}$ and $H \rightarrow \kappa_{1}$ as $N \rightarrow \infty$, we get,

$$
\pi_{k} \sim \eta \kappa_{2} N^{\kappa_{2}-1} x^{\kappa_{2}-1}(1-x)^{\kappa_{1}-1}, N \rightarrow \infty
$$

in which $k \equiv[N x]$ is an integer. By (A.1), we arrive at, 
$\frac{1}{N^{F}} \sum_{k=0}^{N-1} \pi_{k} \sim \frac{F}{N} \sum_{k=0}^{N-1} \eta_{k}\left(\frac{k}{N}\right)^{F-1}\left(1-\frac{k}{N}\right)^{H-1}$

the right hand of which is the Riemann sum approximation of,

$\kappa_{2} \eta \int_{0}^{1} x^{\kappa_{2}-1}(1-x)^{\kappa_{1}-1} d x$

Thus,

$\sum_{k=0}^{N} \pi_{k} \sim N^{\kappa_{2}} \kappa_{2} \eta \int_{0}^{1} x^{\kappa_{2}-1}(1-x)^{\kappa_{1}-1} d x$

So that the resulting density on $[0,1]$ is,

$\frac{\pi_{k}}{\sum_{i=0}^{N} \pi_{i}} \sim \frac{1}{N} \frac{x^{\kappa_{2}-1}(1-x)^{\kappa_{1}-1}}{\int_{0}^{1} x^{\kappa_{2}-1}(1-x)^{\kappa_{1}-1} d x} \sim \frac{x^{\kappa_{2}-1}(1-x)^{\kappa_{1}-1} d x}{\int_{0}^{1} x^{\kappa_{2}-1}(1-x)^{\kappa_{1}-1} d x}$

since $d x \sim 1 / N$. This is a Beta distribution with parameters $\kappa_{1}$ and $\kappa_{2}$.

Note

(1) Although there are some papers such as Jovanovic (1982), Cabral and Mata (2003) and Malerba (2006) study firm's behavior and industrial organization from the evolutionary perspective, little research focuses on evolutionary analysis of cartel formation. And hence the present exploration can be regarded as an effective supplement to this issue.

\section{References}

Bain, J.S. (1951). "Relation of Profit Rate to Industry Concentration: American Manufacturing, 1936-1940", Quarterly Journal of Economics, 65, pp. 293-324

Bain, J.S. (1956). Barriers to New Competition, Cambridge: Harvard University Press

Bain, J.S. (1959). Industrial Organization, John Wiley and Sons, New York

Bos, I. Harrington, J.E. Jr. (2010). "Endogenous Cartel Formation with Heterogeneous Firms", RAND Journal of Economics, 41, pp. 92-117

Cabral, L.M.B., Mata, J. (2003). "On the Evolution of the Firm Size Distribution: Facts and Theory", American Economic Review, 93, pp. 1075-1090 
Compte, O., Jenny, F., Rey, P. (2002). "Capacity Constraints, Mergers and Collusion”, European Economic Review, 46, pp. 1-29

Escrihuela-Villar, M. (2008). "Partial Coordination and Mergers among Quantity-Setting Firms", International Journal of Industrial Organization, 26, pp. 803-810

Escrihuela-Villar, M. (2009). "A Note on Cartel Stability and Endogenous Sequencing with Tacit Collusion", Journal of Economics, 96, pp. 137-147

Ewens, W.J. (2004). Mathematical Population Genetics, 2nd edition. New York: Springer

Fudenberg, D., Imhof, L.A. (2006). „Imitation Process with Small Mutations”, Journal of Economic Theory, 131, pp. 251-262

Fudenberg, D., Imhof, L.A. (2008). "Monotone Imitation Dynamics in Large Populations", Journal of Economic Theory, 140, pp. 229-245

Fudenberg, D., Nowak, M.A., Taylor, C., Imhof, L.A. (2006). "Evolutionary Game Dynamics in Finite Populations with Strong Selection and Weak Mutation", Theoretical Population Biology, 70, pp. 352-363

Imhof, L.A., Nowak, M.A. (2006). "Evolutionary Game Dynamics in a Wright-Fisher Process", Journal of Mathematical Biology, 52, pp. 667-681

Jovanovic, B. (1982). "Selection and the Evolution of Industry", Econometrica, 50, pp. 649-670

Karlin, S., Taylor, H. (1975). A First Course in Stochastic Processes, Academic Press

Levenstein, M., Suslow, V.Y. (2006). "What Determines Cartel Success?", Journal of Economic Literature, 44, pp. 43-95

Malerba, F. (2006). "Innovation and the Evolution of Industries", Journal of Evolutionary Economics, 16, pp. 3-23

Martin, S. (2002). Advanced Industrial Economics, Second edition, Oxford: Blackwell

Nowak, M.A., Sasaki, A., Taylor, C., Fudenberg, D. (2004). "Emergence of Cooperation and Evolutionary Stability in Finite Populations", Nature, 428, pp. 646-650

Prokop, J. (1999). "Process of Dominant-Cartel Formation", International Journal of Industrial Organization, 17 , pp. 241-257

Selten, R. (1973). "A Simple Model of Imperfect Competition, Where 4 Are Few and 6 Are Many", International Journal of Game Theory, 2, pp. 141-201

Stigler, G. (1964). "A Theory of Oligopoly", Journal of Political Economy, 72, pp. 44-61

Sutton, J. (1991). Sunk Costs and Market Structure: Price Competition, Advertising, and the Evolution of Concentration, Cambridge: MIT Press

Sutton, J. (1998). Technology and Market Structure: Theory and History, Cambridge: MIT Press

Symeonidis, G. (2002). The Effects of Competition: Cartel Policy and the Evolution of Strategy and Structure in British Industry, Cambridge: MIT Press

Taylor, C., Fudenberg, D., Sasaki, A., Nowak, M.A. (2004). "Evolutionary Game Dynamics in Finite Populations", Bulletin of Mathematical Biology, 66, pp. 1621-1644

Tirole, J. (1988). The Theory of Industrial Organization, Cambridge: MIT Press

Vasconcelos, H. (2005). "Tacit Collusion, Cost Asymmetries, and Merger", RAND Journal of Economics, 36, pp. 39-62

Williamson, O.E. (1975). Markets and Hierarchies: Analysis and Antitrust Implications, New York: Free Press 